\title{
Kommission Psychologie in Lehramtsstudiengängen
}

\section{Qualitätssicherung schriftlicher Prüfungen nicht nur in der, sondern auch durch die Psychologie}

\author{
Barbara Drechsel ${ }^{1}$, Tobias Dörfler ${ }^{2}$, Kirsten Berthold ${ }^{3}$ und Stephan Dutke ${ }^{4}$ \\ 'Otto-Friedrich-Universität Bamberg \\ ${ }^{2}$ Pädagogische Hochschule Heidelberg \\ ${ }^{3}$ Universität Bielefeld \\ ${ }^{4}$ Westfälische Wilhelms-Universität Münster
}

Die Kommission Psychologie in Lehramtsstudiengängen der DGPs stimmt den Positionen und Forderungen der Autorinnen und Autoren des Plädoyers zur Qualitätssicherung schriftlicher Prüfungen im Psychologiestudium (Lindner et al., 2021) weitgehend zu. Allerdings gilt es, die hier vorherrschende Innenperspektive (gerichtet auf die Prüfungspraxis in der Psychologie) durch eine Außenperspektive (Gestaltung von Prüfungspraxis durch die Psychologie) zu ergänzen. Dies betrifft die Lehramtsstudiengänge in besonderem Maße, weil die Psychologie maßgeblich an ihnen beteiligt ist und Lehramtsstudierende nicht nur Prüfungen ausgesetzt sind, sondern diese später selbst methodisch fundiert konstruieren sollen.

Wie im „Plädoyer“ beschrieben hat der Bologna-Prozess die Anzahl von Prüfungen erhöht und damit die Arbeitsbelastung der Prüfenden. Folglich wurde die Verwendung schriftlicher, geschlossener Formate häufiger. Prüfungen qualitativ hochwertig zu gestalten, ist im Psychologiestudium wie in den Lehramtsstudiengängen von gleichermaßen hoher Bedeutung - im Lehramtsstudium jedoch von ungleich größerer Tragweite, gemessen an der Anzahl der betroffenen Studierenden und ihrer künf- tigen Rolle als Lehrkräfte in unserem Bildungssystem. Der hieraus resultierenden Verantwortung sollte sich die Psychologie als an der Lehrkräftebildung maßgeblich beteiligtes Fach bewusst sein.

Mit seiner Beteiligung an Lehramtsstudiengängen trägt die Psychologie zur Professionalisierung angehender Lehrkräfte bei. Neben der Entwicklung von Lehrkräften als Expertinnen und Experten für das Lernen gehört die psychologische bzw. die pädagogisch-psychologische Diagnostik zu den zentralen Themen, die im Lehramtsstudium in der Psychologie unterrichtet werden (vgl. das Rahmencurriculum für Psychologie in den Lehramtsstudiengängen; DGPs, 2020) und die im Forschungsfeld der Diagnostischen Kompetenz von Lehrkräften in den Blick genommen werden (z.B. Artelt, 2016; Loibl, Leuders \& Dörfler, 2020).

Im Lehramtsstudium wird diagnostisches Wissen zu grundlegenden Fragen und Vorgehensweisen der Diagnostik von Lernvoraussetzungen, Schulleistungen und Lernergebnissen angeboten, aber auch Aufgaben, Materialien oder standardisierte Verfahren fokussiert. Im Kontext des Lehramtsstudiums wird theoriebasiert Wissen 
vermittelt und mit vielfältigen praktischen Anwendungsbeispielen gekoppelt. Beispielsweise können sich angehende Lehrkräfte mit Beurteilungsaufgaben auseinandersetzen, indem sie in einem Seminar eine inhaltlich wie testtheoretisch einwandfrei gestaltete Klausur für ein Unterrichtsfach erstellen. Diese Auseinandersetzung mit Leistungsmessung ist aus der Sicht der Kommission von herausragender Bedeutung, da diagnostische Aufgaben über die gesamte berufliche Laufbahn von Lehrkräften eine zentrale Rolle spielen. Oft entscheiden Lehrkräfteurteile letztlich über Bildungsbiografien. Wie Lehramtsstudierende im Studium geprüft werden, wirkt sich auch auf die Entwicklung von Standards und bestimmter Überzeugungen zu Prüfungen und deren diagnostischer Qualität aus. Qualitativ hochwertige Leistungsdiagnostik im Studium direkt erfahrbar zu machen, sollte deshalb ein wichtiges Ziel im Lehramtsstudium sein. Im Idealfall lernen Studierende auf diese Weise ausgezeichnete Beispiele objektiver, reliabler und (curriculums-)valider Verfahren kennen und können sie als Modell für die spätere eigene diagnostische Praxis nutzen. Doch Lehre in Diagnostik sollte über die reine Modellfunktion hinausgehen. Lehramtsstudierende sollten auf mehreren Ebenen $\mathrm{Zu}$ gang zur Konstruktion von schriftlichen Prüfungen erhalten.

Vermittlung von Vielfalt: Studierende sind insbesondere am Anfang ihres Hochschulstudiums, geprägt durch die Schulerfahrung, hauptsächlich mit offenen Aufgabenformaten vertraut und sind oft skeptisch gegenüber anderen Formaten. Aufgabe der Lehrenden im Fach Psychologie muss es sein, die Vielfalt und Relevanz geschlossener Aufgabenformate transparent zu vermitteln und in Prüfungen anzuwenden. Aufklärungsarbeit ist dabei hinsichtlich unterschiedlicher Gütekriterien - auch der Objektivität und Ökonomie - zu leisten (z.B. Worbach, Drechsel \& Carstensen, 2019). Studierende sollten beispielsweise lernen, dass geschlossene Aufgaben ebenso gut (wenn nicht besser) zwischen leistungsstarken und -schwachen Personen differenzieren können. Der Umstand, dass Lehramtsstudierende in ihren Unterrichtsfächern (und deren Fachdidaktiken) sehr unterschiedliche Fach- und Prüfungskulturen kennenlernen, erhöht die Reichweite psychologisch-diagnostischer Kenntnisse und Methoden, stellt aber auch besondere Herausforderungen. Dazu gehört, dass sich psychologische Forschung (nicht nur im Lehramtsbereich) Anwendungsfragen beim Prüfen nicht-psychologischer Lerngegenstände zuwendet.

„What you test is what you get": Das „Plädoyer" spricht sich im Sinne eines Constructive Alignments für eine hohe Passung von Lehrinhalten, Anforderungen in der Berufspraxis und Prüfungsinhalten in der Hochschule aus. Dem stimmen wir zu. Ergänzend ist das „What you test is what you get"-Prinzip relevant - nicht nur in Bezug auf Lerninhalte, sondern auch hinsichtlich der eingesetzten Lernstrategien. Beispielsweise werden mögliche Klausurfragen zu einem frühen Zeitpunkt einer Vorlesung „Psychologie für die Schule“vorgestellt: ,a) Erläutern Sie für eine Elaborationsstrategie ein eigenes Beispiel, das von den Lehrenden, in der Literatur und im Internet nicht genannt wurde / wird. b) Wie würden Sie aufbauend auf der Forschung zur Förderung von Lernstrategien in Ihrem Unterricht die Anwendung einer Elaborationsstrategie fördern?" Diese Beispielfragen kommunizieren, dass Wissen mit einem hohen Elaborationsgrad und einem Transfer in die Unterrichtspraxis wichtig sind. Idealerweise denken sich daraufhin die Studierenden bereits während des Lernprozesses zu allen Lerninhalten eigene Beispiele aus, verknüpfen so das neue Wissen mit ihrem Vorwissen und wenden die Theorien und Forschungsergebnisse auf ihre Unterrichtspraxis an.

Differenzieren von Messintentionen: Möchte man Studierenden die Messintentionen hinter unterschiedlichen Aufgabentypen näherbringen, müssen neben der Diagnostik auch gedächtnis- und lernpsychologische Inhalte thematisiert werden. Studierenden muss beispielsweise klar werden, welche kognitiven Anforderungen bei der Bearbeitung von geschlossenen Klausuraufgaben gestellt werden. Fehlkonzepten, wie beispielsweise Single- oder Multiple-Choice-Aufgaben erforderten lediglich Wiedererkennen von Gelerntem und seien grundsätzlich nicht zur Reproduktion und Anwendung vernetzter Inhalte geeignet, muss argumentativ entgegengetreten werden. In der Lehre und beim kollegialen Austausch müssen Expertinnen und Experten des Faches gegensteuern - nicht nur bei den Studierenden, sondern teilweise auch bei Vertreterinnen und Vertretern anderer Fächer. Darüber hinaus müssen Lehramtsstudierende aber auch den zielgenauen Umgang mit unterschiedlichen Formaten üben. Während Lindner et al. (2021) mit Blick auf Psychologiestudierende Übung in der Konstruktion eigener Prüfaufgaben als einen „wünschenswerten Nebeneffekt" bewerten, gehört dies bei Lehramtsstudierenden zu deren zentralen Lerninhalten.

Kontextualisierung: Ein grundsätzliches Bedenken gegenüber geschlossenen Aufgabenformate besteht in der möglichen Dekontextualisierung von Lerninhalten. Dabei sind jüngst in unterschiedlichen Kontexten Testformate entstanden, die durch ihre Situiertheit und Kontextualität bei Studierenden und ausgebildeten Lehrkräften auch aufgrund der Augenscheinvalidität mehr Akzeptanz erfahren. Vignettentests etwa sind gute Beispiele dafür, wie man geschlossene Aufgaben sinnvoll in einen didaktisch bedeutsamen Kontext einbetten kann. Lern- und gedächtnispsychologische Forschung bietet vielfältige Anregungen für die Konstruktion derart innovativer Verfahren. 
Zusammengefasst: Welche Aufgaben entstehen vor diesem Hintergrund für die Psychologie?

- Um die skizzierten Lehraufgaben effektiv wahrnehmen zu können, muss die Position der Psychologie im Lehramtsstudium gefestigt und gegebenenfalls ausgebaut werden. Sie muss hinsichtlich ihrer Lehrkapazität so ausgestattet sein, dass sie das von der DGPs geforderte Rahmencurriculum in den Lehramtsstudiengängen jeder Universität und Hochschule auch tatsächlich umsetzen kann.

- Besonders gute Gelegenheiten zur Umsetzung der beschriebenen Lehraufgaben bieten sich in der Begleitung von Praxisphasen, in denen Lehramtsstudierende auch mit Aufgaben im Bereich der Lern- und Leistungsdiagnostik konfrontiert werden. In den meisten Bundesländern liegen diese Praxisphasen auch in der Verantwortung der Hochschulen, weshalb sich Studierenden exzellente Möglichkeiten zur Anwendung psychologisch-diagnostischen Wissens bieten. Insbesondere in der Umsetzung der vielerorts etablierten Studienprojekte im Praxissemester nimmt diese Art des Theorie-Praxis-Transfers eine wichtige Rolle ein (z.B. Souvignier \& Dutke, 2016). Allerdings überlässt die Psychologie die Betreuung der Praxisphasen an vielen Standorten aus kapazitären Gründen anderen Fächern.

- Die Basis all dieser Bemühungen in der Lehre ist pädagogisch-diagnostische Forschung. Nach einer Reihe von Studien, die im Anschluss an die klassische Arbeit von Ingenkamp (1971) entstanden sind, nahmen schulische Leistungsmessung, Notengebung etc. jedoch keine zentrale Rolle mehr in der psychologisch-diagnostischen Forschung ein - ebenso wenig wie neuere Ansätze, wie etwa Lernprozessdiagnostik, Portfolio-Arbeit oder die zuvor erwähnten Vignetten-Tests. Die theoretische und empirische Durchdringung solcher domänenspezifischen Anwendungen auch aus einer grundlagenwissenschaftlichen Perspektive ist notwendig, um sie in den Curricula von Lehramtsstudiengängen nachhaltig zu verankern (vgl. Lindner, Strobel \& Köller, 2015).

Insgesamt ist die Psychologie gefordert, ihre diagnostische Expertise in Richtung schulischer Anwendungen $\mathrm{zu}$ vervollkommnen und sie nicht allein innerhalb der Psychologie, für Prüfungen in den eigenen Studiengängen anzuwenden. Vielmehr sollte sie diese Expertise auch in anderen Studienkontexten zur Verfügung stellen. Lehramtsstudiengänge präsentieren sich hierfür als eine hoch relevante „Zone nächster Entwicklung“. Deshalb stimmen wir den Autorinnen und Autoren des „Plädoyers“ zu, die in der diagnostischen Expertise der Psychologie „eine wesentliche Grundlage für die hochschuldidaktische Aufgabe der Verbesserung von Hochschulprüfungen in unterschiedlichen Fächern" sehen. Nur die Schlussfolgerung dürfte ruhig etwas beherzter ausfallen: Die „Kompetenzen unseres Faches zu bündeln und für eine fächerübergreifende Qualitätssicherung des Prüfungsgeschehens an Hochschulen zur Verfügung stellen“ (Lindner et al., 2021, S. 104) ist nicht nur „(m)öglicherweise [...] eine lohnende Aufgabe“, sondern ganz bestimmt! Vielleicht könnte dieses Wissen eines Tages sogar Einfluss auf Prüfungsordnungen nehmen, die derzeit diagnostischen Fortschritt noch allzu häufig restringieren.

\section{Literatur}

Artelt, C. (2016). Teacher judgments and their role in the educational process: Emerging trends in the social and behavioral sciences: An interdisciplinary, searchable, and linkable Resource. New York, NY: Wiley.

Deutsche Gesellschaft für Psychologie. (2020). Psychologie in den Lehramtsstudiengängen: Ein Rahmencurriculum. Verfügbar unter: https://www.dgps.de/fileadmin/documents/kommissionen/ Rahmencurriculum_Lehramtsstudienga_nge2020.pdf

Ingenkamp, K. (1971). Die Fragwürdigkeit der Zensurengebung. Weinheim: Beltz.

Lindner, M. A., Sparfeldt, J. R., Köller, O., Lukas, J \& Leutner, D. (2021). Ein Plädoyer zur Qualitätssicherung schriftlicher Prüfungen im Psychologiestudium. Psychologische Rundschau, 72, 93 -105. https://doi.org/10.1026/0033-3042/a000524

Lindner, M. A., Strobel, B. \& Köller, O. (2015). Multiple-ChoicePrüfungen an Hochschulen? Ein Literaturüberblick und Plädoyer für mehr praxisorientierte Forschung. Zeitschrift für Pädagogische Psychologie, 29, 133-149. https://doi.org/10.1024/10100652/a000156

Loibl, K., Leuders, T. \& Dörfler, T. (2020). A framework for explaining teachers' diagnostic judgements by cognitive modeling (DiaCoM). Teaching and Teacher Education, 91, 1-10. https:// doi.org/10.1016/j.tate.2020.103059

Souvignier, E. \& Dutke, S. (2016). Studienprojekte im Praxissemester. In E. Jürgens (Hrsg.), Erfolgreich durch das Praxissemester (S. 165-176). Berlin: Cornelsen.

Worbach, M., Drechsel, B. \& Carstensen, C. H. (2019). Messen und Bewerten von Lernergebnissen. In D. Urhahne, M. Dresel \& F. Fischer (Hrsg.), Psychologie für den Lehrberuf (S. 493 -516). Heidelberg: Springer.

Prof. Dr. Barbara Drechsel

Psychologie in Schule und Unterricht

Otto-Friedrich-Universität Bamberg

Markusplatz 3

96047 Bamberg

barbara.drechsel@uni-bamberg.de

https://doi.org/10.1026/0033-3042/a000530 